\title{
Le Flt3-ligand plasmatique, un bio-indicateur pronostique de l'atteinte radio-induite à la moelle osseuse
}

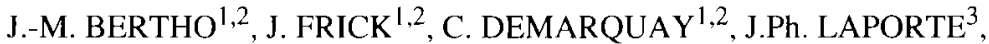 \\ J. AIGUEPERSE ${ }^{4}$, N.-C. GORIN ${ }^{2,3}$, P. GOURMELON ${ }^{2,5}$
}

(Manuscrit reçu le 21 février 200I, accepté le 3 juillet 2001)

RÉSUMÉ À la suite d'une irradiation accidentelle, le choix entre différents traitements du syndrome hématopoïétique dépend essentiellement de l'estimation de l'étendue des dommages radio-induits à la moelle osseuse. Récemment, il a été montré que la concentration dans le sérum du Flt3-ligand (FL), une cytokine agissant sur les cellules immatures de la moelle osseuse, est fortement augmentée lors des aplasies médullaires induites ou acquises. Ceci nous a suggéré que le FL pourrait être un bioindicateur de l'atteinte radio-induite à la moelle osseuse. Afin de vérifier cette hypothèse, un ensemble de travaux a été initié, aussi bien dans un modèle de primate non-humain que chez des patients traités par radio- ou chimiothérapie. Les résultats montrent que la concentration de FL dans le plasma, dans les 5 premiers jours après irradiation, permet de prédire l'évolution du syndrome hématopoḯtique chez l'animal. Des résultats similaires sont retrouvés chez l'homme. L'ensemble de ces études devrait permettre à terme, de disposer d'un bio-indicateur de l'atteinte radioinduite à la moelle osseuse en situation d'irradiation accidentelle.

ABSTRACT Plasma FIt3-ligand as a prognostic bio-indicator of radiation-induced bone marrow damage.

Following an accidental irradiation, the choice between different therapeutic strategies mainly depends upon the estimated radiation-induced bone marrow damage. It was shown recently that the serum concentration of FIt3-ligand (FL), a cytokine mainly acting on immature haemopoietic cells, is highly elevated in either acquired or induced bone marrow aplasia. This suggested to us that FL measurement could be used as a bio-indicator of radiation-induced bone marrow damage. In order to verify this hypothesis, studies were conducted in a non human primate model as well as in humans undergoing radio and/or chemotherapy. Results showed that the increase in FL concentration on day 5 after irradiation is predictive of the evolution of the haemopoietic syndrome. Similar results were obtained in humans. Overall these results suggested that FL measurement could be used as a reliable bio-indicator of radiation-induced bone marrow damage in accidental irradiation situations.

\footnotetext{
'Laboratoire de Radio-hémalologie, IPSN, BP 6, 92265 Fontenay-aux-Roses Cedex, France.

${ }^{2}$ Laboratoire de ther rapie cellulaire el de radioprotection accidentelle. CHU St. Antoine. Paris, France.

${ }^{3}$ Service des maladies du sang, Hôpilal St. Antoine, Paris, France

${ }^{4}$ Section autonome de radiologie appliquée à la médecine, IPSN, BP 6, 92265 Fontenay-aux-Roses Cedex, France.

${ }^{5}$ Département de protection de la santé de l'Homme el de dosimétrie, IPSN, BP 6, 92265 Fontenay-aux-Roses Cedex, France.
} 


\section{La nécessité des bio-indicateurs d'effet de l'irradiation}

L'accident d'irradiation à Tokai-Mura en 1999 (AIEA, 1999) a montré qu'il reste difficile de soigner un irradié accidentel. Cette difficulté est essentiellement due au manque de bio-indicateurs de l'étendue des dommages radio-induits et en particulier des dommages au système hématopoiétique. En effet, le choix entre différents traitements du syndrome hématopoiétique, c'est-à-dire thérapie de soutien, injection de cytokines (Butturini et al., 1988; Mac Vittie et Monroy, 1990 ; Thierry et al., 1995) ou greffe de moelle osseuse (Mathé et al., 1964 ; Baranov et al., 1989 ; Gale et Butturini, 1991), est surtout basé sur l'estimation de la dose reçue par la victime plutôt que sur l'étendue des dommages radio-induits à la moelle osseuse (Gale, 1988). Cependant, l'étude des accidents d'irradiation passés montre clairement que dans la plupart des cas, l'irradiation est hétérogène (Bond et al., 1953 ; Nénot, 1998). Cette hétérogénéité, si elle contre-indique une greffe de moelle osseuse, peut permettre une reconstitution spontanée de la moelle osseuse à partir de territoires médullaires plus ou moins protégés (Scarantino et al., 1984 ; Baranov et al., 1994). L'un des meilleurs exemples reste l'accident de Mol (Belgique) en 1965 (Parmentier et al., 1980), où la victime a reçu des doses estimées de 2 à 3 Gy au niveau de la tête, et jusqu'à 50 Gy au niveau du pied gauche. Malgré un syndrome hématopoḯtique sévère et une dose moyenne au corps entier de $6 \mathrm{~Gy}$, il y a eu une reconstitution spontanée en 3 mois, à partir de $16 \%$ des territoires médullaires irradiés à moins de 3 Gy (Parmentier et al., 1980).

Il apparaît donc clairement qu'en situation d'irradiation accidentelle, il y a un besoin crucial de bio-indicateurs, capables de renseigner spécifiquement sur l'étendue des dommages à un système physiologique spécifique, et en particulier sur l'étendue des dommages à la moelle osseuse. De nombreuses études se sont attachées à définir de tels bio-indicateurs, en utilisant des paramètres biochimiques du sang (Walden et Farzaneh, 1990 ; Greenstock et Trivedi, 1994 ; Donnadieu et al., 1999), la chute des lymphocytes et des polynucléaires du sang périphérique (Dutreix et al., 1987), éventuellement associé à des modèles mathématiques de dynamique des populations (Fliedner et al., 1996), et le dénombrement des anomalies chromosomiques (UNSCEAR, 1988 ; Voisin, 1997). Certaines de ces approches sont très efficaces pour déterminer la dose moyenne à l'organisme. Ainsi, une approche combinée, cytologique et hématologique, a été utilisée avec succès à la suite de l'accident de Tchernobyl (Silini et Gouskova, 1991). Une telle approche multi-paramétrique est d'ailleurs développée par d'autres équipes (Fliedner et al., 2001). Cependant, ces différentes approches se sont révélées décevantes lorsqu'il s'agit de déterminer l'atteinte radio-induite à la moelle osseuse, dans le but de faire des choix thérapeutiques. 


\section{La concentration de Fit3-ligand, bio-indicateur d'atteinte radio-induite à la moelle osseuse ?}

L'aplasie médullaire radio-induite est due à la disparition de la majorité des cellules souches et immatures de la moelle osseuse. La reconstitution hématopoïetique est donc directement corrélée à la capacité des cellules souches résiduelles à proliférer et à se différencier pour générer les cellules matures et fonctionnelles du sang périphérique. La prolifération et la différenciation des cellules souches sont régulées par différents mécanismes, parmi lesquels les cytokines jouent un rôle important. L'une d'entre elle, le Flt3-ligand (FL), a été clonée à partir de lignées de cellules lymphoïdes (Lyman et al., 1994 ; Hannun et al., 1994). Le FL agit essentiellement sur la prolifération des cellules souches et des progéniteurs du système hématopoiétique (Fichelson, 1998), soit seul (Lyman et al., 1994), soit en combinaison avec d'autres cytokines comme le Stem Cell Factor (SCF) (Ray et al., 1996). Le FL agit également sur les précurseurs lymphoïdes B (Hunte et al., 1995) et sur les précurseurs lymphocytaires $\mathrm{T}$ intra-thymiques (Bertho et al., 2000).

Il a été montré que la concentration sérique de FL est fortement augmentée chez les patients qui souffrent d'anémie de Fanconi ou d'anémie médullaire idiopathique (Lyman et al., 1995), deux pathologies d'aplasie médullaire spontanée. D'autre part, il a été montré qu'une forte élévation de la concentration de FL dans le sang apparaît à la suite de traitements aplasiants, comme la chimiothérapie (Wodnar-Filipowicz et al., 1996). Ces mêmes auteurs ont montré que la concentration de $\mathrm{FL}$ dans le sang périphérique est corrélée négativement à la fréquence des progéniteurs présents dans la moelle osseuse (Wodnar-Filipowicz et al., 1996). Étant donné que l'aplasie radio-induite est essentiellement due à la disparition des cellules souches et progéniteurs médullaires de la moelle osseuse, nous avons posé l'hypothèse que le suivi de la concentration de FL dans le sang périphérique pourrait être utilisé en tant que bio-indicateur de l'atteinte radioinduite à la moelle osseuse en situation d'irradiation accidentelle.

\section{3. Études chez le primate non-humain : un modèle pré-clinique}

Une étude a été initiée chez le primate non-humain irradié de façon à vérifier cette hypothèse. Tout d'abord, le niveau de base du FL a été mesuré chez 24 animaux, dont trois femelles, au minimum deux fois par animal à au moins deux mois d'intervalle. Ceci a permis de vérifier qu'il est possible de doser le FL de primate avec un test de détection de la cytokine humaine, mais également de définir un niveau de base de $131,9 \pm 36 \mathrm{pg} / \mathrm{ml}$. De plus, aucune variation significative du niveau du FL n'a été détectée en fonction de l'âge et du sexe des animaux, ni en fonction du cycle circadien (Fig. 1) (Bertho et al., 2001). 


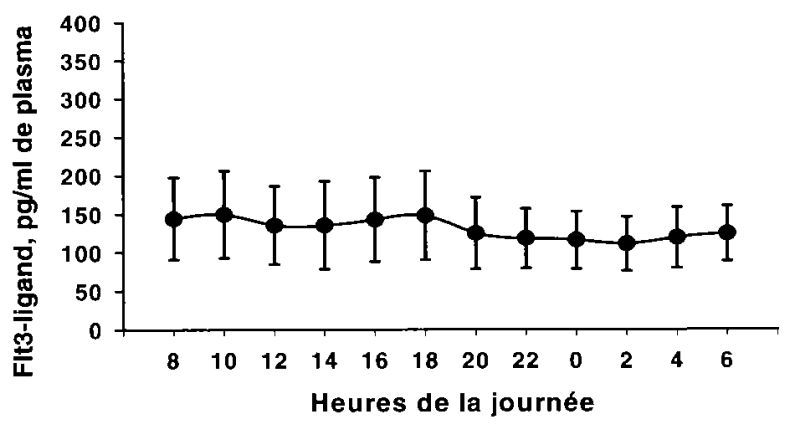

Figure 1 - Variations de la concentration de FL en fonction du cycle circadien. L'étude a été faite chez 4 animaux ( 2 mâles et 2 femelles) sur une période de 36 heures. Chaque point représente la moyenne ( \pm écart-type) de l'ensemble des mesures faites à la même heure sur les 4 animaux. Aucune variation significative en fonction du cycle circadien n'est observée.

Variations of plasma FL concentration according to the circadian rhythm. The study was made with 4 animals ( 2 males and 2 females) during 36 hours. Results are the mean ( $\pm S E$ ) of all measurements made at the same hour. No significant variation according to the circadian rhythm was observed.

Différentes expériences d'irradiation de primates ont été réalisées, et ont permis de suivre l'évolution de la concentration plasmatique du FL en fonction de la configuration d'irradiation (homogène ou hétérogène), de la dose d'irradiation (de 2 à $8 \mathrm{~Gy}$ ), ou du traitement du syndrome hématopoiétique mis en place. Les résultats montrent que chez tous les animaux irradiés, il y a une élévation de la concentration plasmatique de FL dans la première semaine qui suit l'irradiation. Cette élévation apparaît dès le $2^{\mathrm{e}}$ jour, avant même les modifications du nombre de neutrophiles dans le sang périphérique, et se poursuit jusqu'à un pic, qui précède le nadir des neutrophiles (Bertho et al., 2001). Il est possible de mettre en évidence, dès le $5^{\mathrm{e}}$ jour après une irradiation homogène, une relation entre la dose d'irradiation et l'augmentation de la concentration du FL (Fig. 2). En effet, Les animaux irradiés à des doses inférieures à la dose létale $50\left(\mathrm{DL}_{50}\right)$ (estimée à 6 Gy chez M. Fascicularis) (Zoetelief et al., 1998) ont une augmentation de concentration du FL d'un facteur 6 au maximum, alors que les animaux irradiés à des doses létales ou supra létales ont une augmentation de la concentration de FL supérieure à un facteur 10 dans les 5 premiers jours après irradiation (Fig. 2). Il est donc possible de distinguer, sur la base de la concentration plasmatique de FL au $5^{\mathrm{e}}$ jour après irradiation homogène, les animaux irradiés à doses infra-létales des animaux irradiés à doses supra-létales. Par la suite, la diminution de la concentration plasmatique de FL et son retour à la normale précède la sortie d'aplasie et le retour à la normale de la numération et de la formule sanguine. 


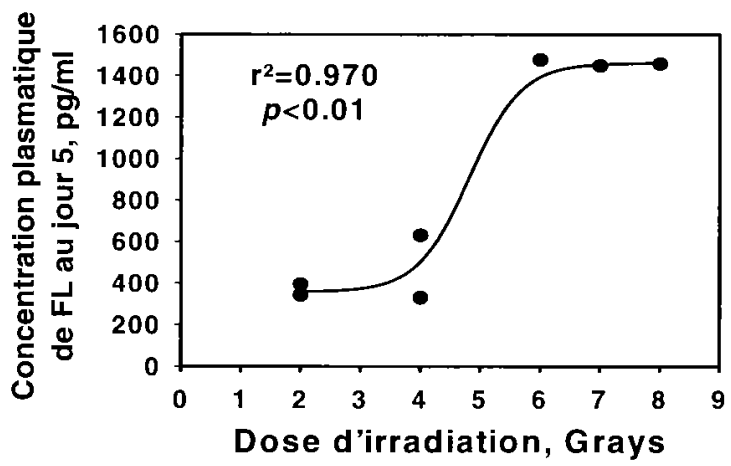

Figure 2 - Analyse de corrélation entre la valeur de la concentration plasmatique de FL au $5^{e}$ jour après irradiation et la dose d'irradiation pour les animaux non-traités pour le syndrome hématopoḯtique. Chaque point représente la valeur individuelle de chaque animal ayant reçu une irradiation corporelle totale.

Correlation analysis between day 5 plasma FL concentration on day 5 post-irradiation and irradiation dose for untreated animals. Each point represent individual value for each animal receiving a total body irradiation.

Les variations de la concentration plasmatique de FL précèdent également les variations du nombre de plaquettes circulantes (Bertho et al., 2001). En effet, l'augmentation de la concentration de FL précède de 3 à 13 jours le début de la thrombopénie, qui elle-même est directement corrélée à la dose d'irradiation. De plus, le retour à la normale de la concentration de FL précède également la normalisation du nombre de plaquettes circulantes. Par contre, l'augmentation de concentration de FL ne permet pas de prédire la profondeur de la thrombopénie. Ceci est lié au fait que les animaux thrombopéniques sont régulièrement transfusés avec des culots plaquettaires (de façon à éviter les hémorragies), ce qui modifie le nombre de plaquettes circulantes.

Le suivi des variations de la concentration du FL permet donc de prédire les variations de la numération et de la formule sanguine, et donc de prédire la gravité de l'atteinte radio-induite à la moelle osseuse. En effet, nous avons pu mettre en évidence un ensemble de corrélations entre la concentration plasmatique de FL au $5^{\mathrm{e}}$ jour après irradiation et différents paramètres de la neutropénie et de la thrombopénie (Tab. I) lorsque les animaux reçoivent uniquement un traitement de support constitué d'antibiotiques et de transfusions de plaquettes (animaux qualifiés par la suite de «non-traités »). Ces corrélations permettent de prédire le temps de latence (intervalle de temps entre l'irradiation et la chute à moins de $0,5 \times 10^{9}$ neutrophiles par litre de sang), la durée d'aplasie (temps durant lequel les neutrophiles sont à moins de $0,5 \times 10^{9}$ par litre de sang), et la profondeur du nadir, 
TABLEAU I

Récapitulatif des corrélations obtenues entre la concentration en FL à 5 jours post-irradiation et différents paramètres de l'aplasie radio-induite, en fonction de la présence ou non d'un traitement du syndrome hématopoïétique. Les animaux sans traitement reçoivent uniquement une antibiothérapie et des transfusions de plaquettes.

Summary of correlations between day 5 post-irradiation plasma FL concentration and some parameters of radio-induced aplasia, according to the use of a treatment for hematopoietic syndrome. Animals without specific treatment received only antibiotic treatment and platelet transfusions.

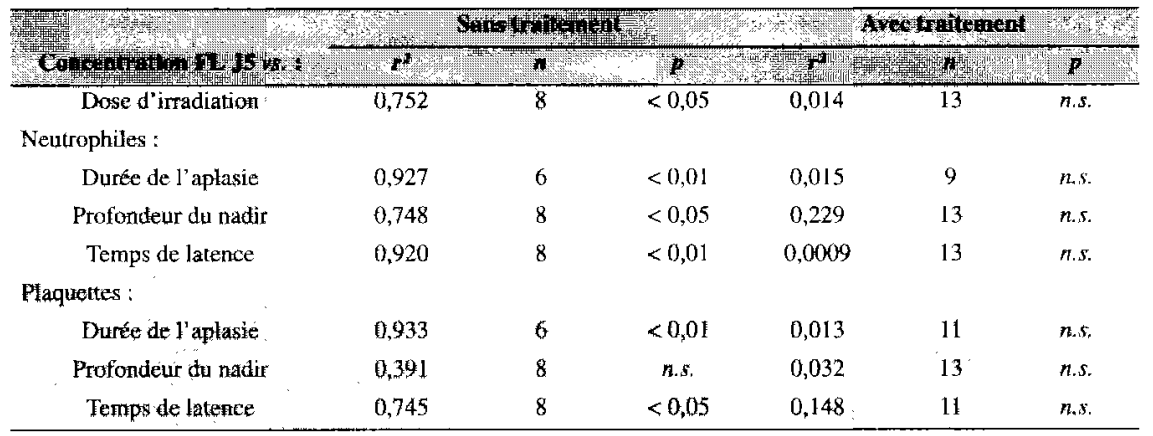

pour les neutrophiles, mais aussi pour les plaquettes (Tab. I). Ceci montre que l'augmentation de la concentration plasmatique de FL au $5^{\mathfrak{e}}$ jour après irradiation permet de prédire la gravité de l'atteinte radio-induite à la moelle osseuse, chez les animaux non-traités, et que le suivi du FL constitue un bio-indicateur de pronostic de l'atteinte radio-induite à la moelle osseuse.

Par contre, les corrélations observées chez les animaux non-traités disparaissent chez les animaux traités par greffe autologue de cellules mononucléées ou de cellules $\mathrm{CD} 34^{+}$de moelle osseuse, ou encore par injection de cytokines (Tab. I). Ceci est lié à la combinaison de deux éléments. Tout d'abord, les traitements par greffe de cellules ou par injection de cytokines modifient l'évolution du syndrome hématopoïétique en réduisant la durée et la profondeur de 1'aplasie, sans modifier le taux de FL au $5^{\mathrm{e}}$ jour après irradiation. Ceci implique que, dans ce cas, la concentration de FL peut être considérée comme un bioindicateur du dommage initial à la moelle osseuse. D'autre part, certains traitements, et en particulier les traitements par cytokines, jouent non seulement sur l'évolution du syndrome hématopoiétique, mais modifient également les valeurs de concentration plasmatique du FL dès la première semaine après l'irradiation. Ceci est probablement lié au fait que les cytokines influent directement sur l'activation des cellules hématopoiétiques, c'est-à-dire sur la dynamique des populations résiduelles après irradiation, et en particulier des 


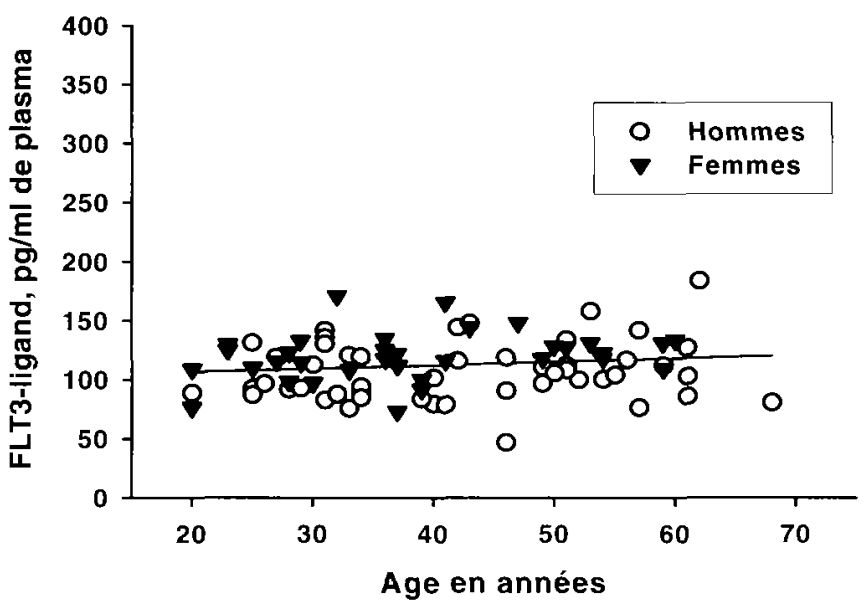

Figure 3-Niveau de FL plasmatique chez l'homme en situation physiologique normale, en fonction de l'âge. Chaque point représente une mesure individuelle. Une différence significative est observée dans la valeur moyenne du FL en fonction du sexe (Hommes : 107,1 $\pm 25,2$ pg/ml, $n=49$, -)- femmes : $121,3 \pm 19,4 \mathrm{pg} / \mathrm{ml}, n=33,-\nabla-p<0,01)$. Par contre, aucune variation signification n'est observée en fonction de l'âge $\left(r^{2}=0,021, n=82\right)$.

Plasma FL concentration in humans in normal physiological situation. Each point represent an individual measurement. A significant difference in plasma $F L$ concentration according to the sex of donors was observed (males: $107.1 \pm 25.2 \mathrm{pg} / \mathrm{ml}, \quad n=49, \quad-)-$; females: $121.3 \pm 19.4 \mathrm{pg} / \mathrm{ml}, n=33,-\nabla-p<0.01)$. By contrast, no significant variation according to the age of donors was observed $\left(r^{2}=0.021, n=82\right)$.

cellules capables de consommer le FL. Il sera donc nécessaire, pour une application future du suivi du FL comme bio-indicateur de l'atteinte à la moelle osseuse, de tenir compte de l'influence des traitements mis en œuvre chez la victime d'irradiation accidentelle.

\section{Transposition à l'homme des résultats obtenus chez l'animal}

La transposition à l'homme des résultats obtenus chez le primate doit être faite avec précaution. Cependant, différentes études ont d'ores et déjà montré que le niveau de base du FL est similaire, malgré la barrière d'espèce. Une étude de notre laboratoire, faite sur 82 volontaires sains ( 33 femmes et 49 hommes; âge médian : 36 ans, extrêmes : 20-68 ans), a montré une moyenne de $112,8 \pm 24 \mathrm{pg} / \mathrm{ml}$ de plasma (47-183,8 pg/ml, voir Fig. 3). Le niveau de FL chez les hommes est légèrement plus faible que chez les femmes $(107,1 \pm 25,2 \mathrm{pg} / \mathrm{ml}$ vs. $121,3 \pm$ $19,4 \mathrm{pg} / \mathrm{ml}$ respectivement, $p<0.01$ ) et aucune variation du niveau de FL n'est observée en fonction de l'âge (Laporte et al., 2000). D'autres équipes ont montré 


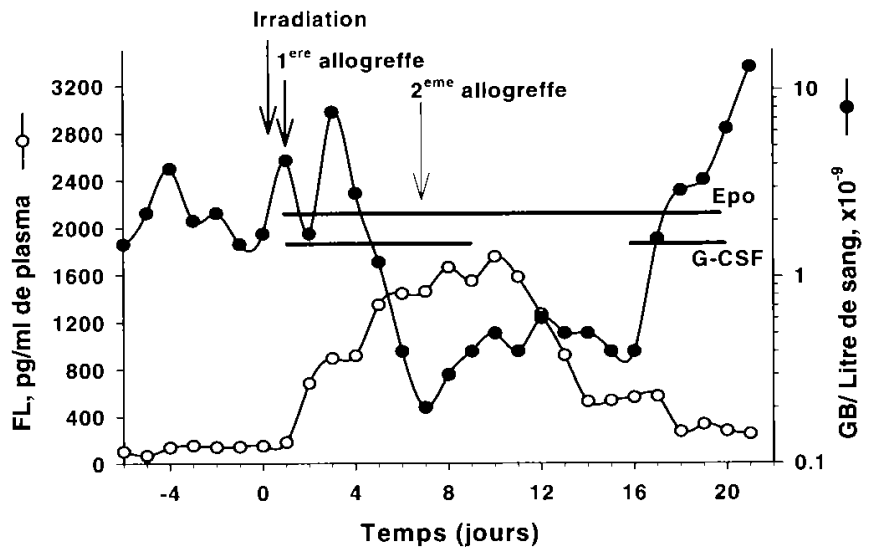

Figure 4 - Suivi comparé du nombre de globules blancs (GB) circulants (-O) et de la concentration plasmatique de FL (-) chez un patient représentatif souffrant de leucémie aiguë lymphoblastique et traité par irradiation corporelle totale et allogreffe de moelle osseuse. Les variations de la concentration plasmatique du FL précèdent toujours les variations du nombre de globules blancs.

Comparative evolution of white blood cell numbers (GB, -0-) and plasma FL concentration (-)-) in a representative patient with an acute lymphoblastic leukemia and treated with total body irradiation and bone marrow allograft. Plasma FL concentration variations always appeared before white blood cell number variations.

des taux sériques de FL similaires, compris entre $53 \mathrm{pg} / \mathrm{ml}$ (Todd et al., 1997) et $94 \mathrm{pg} / \mathrm{ml}$ (Blumenthal et al., 2000). Ces taux légèrement plus faibles peuvent s'expliquer par le fait que nos mesures ont été faites dans le plasma.

Comme indiqué précédemment, il a été montré par d'autres auteurs que le niveau de FL est augmenté chez les patients traités par chimiothérapie (WodnarFilipowicz et al., 1996). Nous avons cherché à étendre ces observations à une plus grande variété de patients, incluant en particulier des patients traités par irradiation corporelle totale avant une greffe de moelle osseuse. Les premiers résultats de cette étude montrent que, d'une façon générale, les résultats obtenus chez le primate non-humain sont retrouvés chez les patients irradiés en corporel total.

En effet, une augmentation du taux plasmatique de FL est observée dans la première semaine après l'irradiation chez les 7 patients ayant reçu un traitement aplasiant (chimiothérapie ou radiothérapie) actuellement étudiés (Fig. 4, résultats obtenus chez un patient irradié). La courbe d'évolution est alors inversée par rapport à la courbe de suivi des globules blancs dans le sang périphérique, et la diminution du taux de FL précède de 2 à 5 jours la remontée du nombre de globules blancs, quels que soient le patient, la pathologie ou le traitement appliqué. Bien 
que ces résultats soient très préliminaires, ils montrent que les variations du FL après traitement aplasiant sont très proches de ce qui a été observé chez le primate non-humain, et donc qu'une extrapolation des résultats du modèle primate vers l'homme est possible. Cependant, pour que le suivi du FL puisse être appliqué au cas des irradiations accidentelles, il est nécessaire de mieux comprendre les mécanismes de régulation de la production de cette cytokine.

\section{Les mécanismes de régulation du Flt3-ligand}

Les mécanismes de régulation de la concentration de FL dans le sang périphérique, et en particulier après irradiation, restent largement inconnus. Le FL est produit de façon ubiquitaire dans l'organisme (Fichelson, 1998). Une hypothèse est que la régulation du niveau de FL dans le sang se fait par consommation de la cytokine par les cellules porteuses du récepteur, le Flk2/Flt3 (Fichelson, 1998). De fait, l'expression du récepteur au FL (FLr) est restreinte aux cellules souches hématopoiétiques et aux cellules immatures du système lympho-hématopoiétique (Rosnet et al., 1993 ; Small et al., 1994 ; Bertho et al., 2000). Selon cette hypothèse, les variations du niveau de FL dans le sang périphérique après traitement myéloablatif dépendent essentiellement de la disparition et de la reconstitution des cellules hématopoiétiques. Cette hypothèse permet d'expliquer l'effet prédictif des variations du niveau de FL par rapport à l'évolution de la numération/formule sanguine. Elle permet également d'expliquer la perte de prédictivité de la concentration plasmatique de FL à J5 à la suite d'une greffe de moelle osseuse, du fait de l'apport de cellules capables de consommer le FL. Cependant, Chklovskaia et al. (1999) ont montré que les lymphocytes $\mathrm{T}$ du sang périphérique produisent et stockent du FL dans le réticulum endoplasmique. D'autre part, les mêmes auteurs ont montré qu'à la suite d'un traitement myéloablatif, le stock de FL est rapidement transporté jusqu'à la surface de la cellule où la cytokine est exprimée sous forme membranaire avant d'être relâchée dans la circulation. Un calcul théorique permet aux auteurs d'affirmer que la quantité de FL produite par les lymphocytes T est suffisante pour expliquer l'augmentation initiale de la concentration de FL dans le plasma (Chklovskaia et al., 1999). Un argument en faveur de cette hypothèse est que, dans notre étude de suivi des patients recevant une greffe de moelle osseuse, nous avons observé une diminution de la concentration plasmatique de FL chez les patients recevant un traitement par sérum anti-lymphocytaire en plus de la chimiothérapie ou de la radiothérapie (Laporte et al., 2000). Cependant, il est bien connu que l'irradiation fait chuter très rapidement le nombre de lymphocytes circulants (Dutreix et al., 1987). Or, malgré cette déplétion en lymphocytes, un niveau élevé de FL est observé jusqu'à la sortie d'aplasie. Il est donc possible que d'autres types cellulaires puissent produire du FL, et en particulier les cellules des microenvironnements médullaires et thymiques. En effet, il a été démontré une 
expression de l'ARNm dans les cellules des micro-environnements médullaires et thymiques (Hannum et al., 1994 ; Lyman et al., 1994), et une production de FL par les cellules stromales de moelle osseuse (Lisovsky et al., 1996). Une augmentation de l'expression des ARN messagers (ARNm) codant pour le FL dans le thymus et dans la moelle osseuse après un traitement myéloablatif a aussi été mise en évidence (Chklovskaia et al., 1999). Plus récemment, une équipe (Solanilla et al., 2000) a démontré la production de FL par les cellules endothéliales. Cet ensemble de données suggère que les lymphocytes $\mathrm{T}$ ne sont pas les seuls responsables de l'augmentation de la concentration du FL dans les situations d'aplasie médullaire, et que les mécanismes de régulation du niveau de FL dans le sang périphérique sont plus complexes qu'une simple régulation par consommation comme initialement suggéré (Ficheslon, 1998).

\section{Le Flt3-ligand présente toutes les caractéristiques d'un bon bio-indicateur}

Les résultats présentés ici montrent clairement que le suivi de la concentration de FL pourrait être utilisé comme bio-indicateur de pronostic de l'atteinte radioinduite à la moelle osseuse. De plus, la mesure du FL répond à toutes les caractéristiques nécessaires pour l'application à la situation d'irradiation accidentelle (Greenstock et Trivedi, 1994) : la prise d'échantillon est de petit volume (1 $\mathrm{ml} \mathrm{de}$ sang prélevé sur EDTA est suffisant), facile à prélever, et ne provoque pas de stress notable. La mesure de la concentration du FL dans le sang périphérique par ELISA est complètement reproductible, facilement automatisable, et rapide à obtenir. Les résultats du laboratoire montrent qu'il n'y a pas de variation en fonction du cycle circadien chez l'animal (Bertho et al., 2001), et qu'il n'y a pas de variation notable du niveau de FL en fonction de l'âge aussi bien chez l'animal (Bertho et al., 2001) que chez l'homme (Laporte et al., 2000). Une petite différence a été observée en fonction du sexe chez l'homme, mais le niveau de FL en situation physiologique normale est très bas ; le rapport signal/bruit est très élevé et permet de négliger cette différence du niveau de base en fonction du sexe. Enfin, à notre connaissance, il n'existe pas d'autre pathologie humaine autre que les aplasies médullaires spontanées (comme l'anémie de Fanconi ou l'aplasie médullaire idiopathique) (Wodnar-Filipowicz et al., 1996) ou induites (comme les traitements myéloablatifs tels que chimiothérapie ou radiothérapie) (Chklovskaia et al., 1999 ; Laporte et al., 2000) dans lesquelles il existe un niveau élevé de FL dans le sang. Il en résulte que l'augmentation de FL dans le sang périphérique est spécifique d'une atteinte radio-induite à la moelle osseuse, qu'il y a une relation directe entre vitesse d'augmentation de la concentration du FL et dose d'irradiation reçue (Bertho et al., 2001), et que l'élévation du niveau de FL perdure tant que la moelle osseuse n'a pas reconstitué une activité hématopoiétique normale (Blumenthal et al., 2000 ; 
Bertho et al., 2001). Ceci suggère que le dosage du FL pourrait servir non seulement de bio-indicateur de l'atteinte radio-induite à la moelle osseuse, mais également de bio-indicateur de suivi des greffes de moelle osseuse en clinique humaine, et d'indicateur de diagnostic pour la détection des anémies ou aplasies d'origine médullaire telles que les anémies de Fanconi ou les aplasies médullaires idiopathiques.

La validation du FL en tant que bio-indicateur de l'atteinte radio-induite à la moelle osseuse chez l'homme passe par la poursuite des études en clinique humaine. D'autre part, étant donné que la majorité des irradiations accidentelles sont hétérogènes, il est nécessaire de préciser l'évolution du niveau de FL dans les situations d'irradiation hétérogène. Pour ceci, une étude rétrospective de primates irradiés dans différentes configurations d'irradiation hétérogène est actuellement en cours. D'autre part, une étude va être initiée pour suivre le niveau de FL chez des patients irradiés de façon hétérogène ou localisée. Cette étude devrait permettre à terme de définir si le suivi du FL, que ce soit sous forme soluble dans le sang périphérique ou sous forme membranaire à la surface des lymphocytes $\mathbf{T}$, pourrait être utilisé non seulement comme bio-indicateur de l'atteinte radio-induite à la moelle osseuse, mais également comme marqueur de l'hétérogénéité de l'irradiation.

Remerciements. Les auteurs tiennent à remercier R. Rioux et son équipe pour les soins aux animaux, E. Soler pour son assistance administrative, et l'aide précieuse de $Q$. Chau et de F. Trompier pour la dosimétrie des irradiations. Le regard critique sur le manuscrit de M.H. Gaugler est grandement remercié.

\section{RÉFÉRENCES}

Baranov A., Gale R.P., Guskova A., Piatkin E., Selidovkin G., Muravyova L., Champlin R.E., Danilova N., Yevseeva L., Petrosyan L., Pushkareva S., Konchalovsky M., Gordeeva A., Protasova T., Reisner Y., Mickey M.R., Terasaki P.I. ( 1989) Bone marrow transplantation after the Chernobyl nuclear accident, New Engl. J. Med. 321, 205-212.

Baranov A., Selidovkin G., Butturini A., Gale R.P. (1994) Hematopoietic recovery after 10-Gy acute total body radiation, Blood $\mathbf{8 3}, 596-599$.

Bertho J.M., Chapel A., Loilleux S., Frick J., Aigueperse J., Gorin N.C., Gourmelon P. (2000) CD 135 (Flk2/Flt3) expression by human thymocytes delineates a possible role of Flt3-ligand in $\mathrm{T}$ cell precursor proliferation and differentiation, Scand. J Immunol. 52, 53-61.

Bertho J.M., Demarquay C., Frick J., Joubert C., Arenales C., Jacquet N., Sorokine-Durm I., Chau Q., Lopez M., Aigueperse J., Gorin N.C., Gourmelon P. (2001) Level of Flt3-ligand in plasma: a new bio-indicator for radiation-induced aplasia, Int. J Radiat. Biol. 77, 703-712.

Blumenthal R.D., Lew W., Juweid M., Alisauskas R., Ying Z., Goldenberg D. (2000) Plasma FLT3-L levels predict bone marrow recovery from myelosuppressive therapy, Cancer 88, 333-343.

Bond V.P., Cronkite E.P., Sondhaus O.A. (1953) The influence of exposure geometry on the pattern of radiation dose delivered to large animal phantoms, Radiat. Res. 6, 554-572.

Butturini A., Gale R.P., Lopez D.M. (1988) Use of recombinant granulocyte-macrophage colonystimulating factor in the brazil radiation accident, The Lancet $\mathbf{8 2 7}, 471-474$. 
Chklovskaia E., Jansen W., Nissen C., Lyman S.D., Rhaner C., Landmann L., Wodnar-Filipowicz A. (1999) Mechanism of flt3 ligand expression in bone martow failure: Translocation from intracellular stores to the surface of T-lymphocytes after chemotherapy-induced suppression of hematopoiesis, Blood 93, 2595-2603.

Dutreix J., Girinsky T., Cosset J.M., Bernard A., Pico J., Baume D., Bayle Ch., Benk V. (1987) Blood cell kinetics and total body irradiation, Radiother. Oncol. 9, 119-129.

Donnadieu-Claraz M, Benderitter M., Joubert C., Voisin P. (1999) Biochemical indicator of wholebody $\gamma$-radiation effects in the pig, Int. J. Radiat. Biol. 75, 165-174

Fichelson S. (1998) The FLT3/FLK2 ligand: structure, functions and prospects, Eur. Cytokine Network 9, 7-22.

Fliedner T.M., Tibken B., Hofer E.P., Paul W. (1996) Stem cell response after radiation exposure: A key to the evaluation and prediction of its effects, Health Phys. 70, 787-797.

Fliedner T.M., Friesecke I., Beyrer K. (Eds.) (2001) Medical management of radiation accidents, British Institute of Radiology, London.

Gale R.P. (1988) Perspective - Medical response to radiation and nuclear accidents: lessons for the future, J. Natl. Cancer Inst. 80, 995-998.

Gale R.P., Butturini A. (1991) Medical response to nuclear and radiation accidents, Occup. Med. 6, $581-596$

Greenstock C.L., Trivedi A. (1994) Biological and biophysical techniques to assess radiation exposure: A perspective, Prog. Biophys. Molec: Biol. 61, 81-130.

Hannum C., Culpepper J., Campbell D., McClanahan T., Zurawski S., Bazan J.F., Kastelein, R., Hudak S., Wagner J., Mattson J., Luh J., Duda G., Martina N., Peterson D., Menon S., Shanafelt A., Muench N., Kelner G., Namikawa R., Rennick D., Roncarolo M.-G., Zlotnik A., Rosnet O., Dubreuil P., Birnbaum D., Lee F. (1994) Ligand for FLT3/FLK2 receptor tyrosine kinase regulates growth of haematopoietic stem cells and is encoded by variant RNAs, Nature $\mathbf{3 6 8}$, 643-648.

Hunte B.E., Hudak S., Campbell D., Xu Y., Rennick D. (1995) Flk2/Flt3 ligand is a potent cofactor for the growth of primitive B cell progenitors, J. Immunol. 156, 489-496.

International Atomic Energy Agency (1999) Report on the preliminary fact finding mission following the accident at the nuclear fuel processing facility in Tokaimura, Japan (Vienna, Austria).

International Atomic Energy Agency (1986) Biological dosimetry: chromosomal aberrations analysis for dose assessment, Techn. Rep. 260 (Vienna, Austria).

Jilma B., Hergovich N., Stohlawetz P., Eichler H.G., Bauer P., Wagner O.F. (1999) Circadian variation of granulocyte colony stimulating factor levels in man, British J. Haematol. 106, 368-370.

Laporte J.P., Bertho J.M., Frick J., Demarquay C., Joubert C., Lopez M., Gorin N.C., Gourmelon P. (2000) Flt3-ligand (Flt3-1) plasma level as a predictive indicator of bone marrow recovery after high dose chemotherapy (HDCT) and/or total body irradiation (TBI) preconditionning regimen, Blood 96, 394a.

Lisovsky M., Braun S.E., Ge Y., Takahira H., Lu L., Savchenko V.G., Lyman S.D., Broxmeyer H.E. (1996) Flt3-ligand production by human bone marrow stromal cells, Leukemia 10, 1012-10.

Lyman S.D., James L., Johnson L., Brasel K., De Vries P., Escobar S.S., Downey H., Splett R.R., Beckmann M.P., McKenna H.J. (1994) Cloning of the human homologue of the murine Flt 3 ligand: A growth factor for early hematopoietic progenitor cells, Blood 83, 2795-2801.

Lyman S.D., Seaberg M., Hanna R., Zappone J., Brasel K., Abkowitz J.L., Prchal J.T., Schultz J.C., Shahidi, N.T. (1995) Plasma/Serum levels of Flt3 ligand are low in normal individuals and highly elevated in patients with Fanconi anemia and acquired aplastic anemia, Blood 86, 40914096.

MacVittie T.J., Monroy R.L. (1990) Potential improvement in the management of seriously irradiated persons, in: The medical basis for radiation accident preparedness, R.C. Ricks, S.A. Fry (Eds.) Elsevier Science Publishing Co., pp. 121-147. 


\section{LE FLT3-LIGAND PLASMATIQUE}

Mathé G., Amiel J.L., Schwarzenberg L. (1964) Treatment of acute total body irradiation injury in man, Ann. N.Y. Acad. Sciences 114, 368-392.

Namikawa R., Muench M.O., Roncarolo M.G. (1996) Regulatory roles of the ligand for Flk2/Flt3 tyrosine kinase receptor on human hematopoiesis, Stem Cells 14, 388-395.

Nénot J.C. (1998) Radiation accidents: Lessons learnt for the future radiological protection, Int. $J$. Radiat. Biol. 73, 435-442.

Parmentier N.C., Nénot J.C., Jammet H.J. (1980) A dosimetric study of the belgian (1965) and the italian (1975) accidents, in: The medical basis for radiation accident preparedness, K.F. Hübner, S.A. Fry (Eds.) Elsevier Science Publishing Co., pp. 105-112.

Ray R.J., Paige C.J., Furlonger C., Lyman S.D., Rottapel R. (1996) Flt3 ligand supports the differentiation of early $B$ cell progenitors in the presence of interleukin- 11 and interleukin-7, Eur. J. Immunol. 26, 1504-1510.

Rosnet O., Schiff C., Pébusque M-J., Marchetto S., Tonnelle C., Toiron Y., Birg F., Birnbaum D. ( I 993) Human Flt3/Flk2 gene: cDNA cloning and expression in hematopoietic cells, Blood $\mathbf{8 2}$ $1110-1119$.

Rusten L.S., Lyman S.D., Veiby O .P., Jacobsen S.E. (1996) The Flt3 ligand is a direct and potent stimulator of the growth of primitive and committed human CD34 $4^{+}$bone marrow progenitor cells in vitro, Blood 87, 1317-1325.

Scarantino C.W., Rubin P., Constine L.S. (1984) The paradoxes in patterns and mechanisms of bone marrow regeneration after irradiation. I. Different volumes and doses, Radiother. Oncol. 2, 215-225.

Silini G., Gouskova A. (1991) Biological dosimetry at Chernobyl, in: New horizons in biological dosimetry, Wiley-Liss, New-York, pp. 129-144.

Small D., Levenstein M., Kim E., Carow C., Amin S., Rockwell P., Witte L., Burrow C., Ratajczak M.Z., Gewirtz A.M., Civin C.I. (1994) STK-1, the human homolog of Flk-2/Flt-3, is selectively expressed in $\mathrm{CD}_{34}{ }^{+}$human bone marrow cells and is involved in the proliferation of early progenitor/stem cells, Proc. Natl. Acad. Sci. USA 91, 459-463.

Solanilla A., Grosset C., Lemercier C., Dupouy M., Mahon F.X., Schweitzer K., Reiffers J., Weksler B., Ripoche J. (2000) Expression of Flt3-ligand by the endothelial cell, Leukemia 14, 153-162.

Sonoda Y., Kimura T., Sakabe H., Tanimukai S., Ohmizono Y., Nakagawa S., Yokota S., Lyman S.D., Abe T. (1997) Human Flt3 ligand acts on myeloid as well as multipotential progenitors derived from purified $\mathrm{CD} 34^{+}$blood progenitors expressing different levels of $c$-kit protein, Eur. $J$. Haematol. 58, 257.

Thierry D., Gourmelon P., Parmentier C., Nenot J.C. (1995) Haematopoietic growth factors in the treatment of therapeutic and accidental irradiation-induced bone marrow aplasia, Int. J. Radiat. Biol. 67, 103-117.

Todd G., Haug J.S., Bremer R., Link D., Goodnough L., Adkins D., Brown R., DiPersio J. (1997) Flt3ligand levels vary before and during G-CSF mobilization, Blood 10 (Sup. 1), 211 a.

UNSCEAR (1988) Sources, effects and risks of ionizing radiations, appendix G, pp. 207-287.

Voisin P. (1997) Chromosome lesions as a short and medium term biological indicator of acute irradiation, L'Homme blessé, Court L.A., Lallemand J. (Eds.), CRSSA, EDF, IPSN, Jouve France $\left(\mathrm{N}^{\circ} 248935 \mathrm{M}\right)$, pp. 137-150.

Walden T.L., Farzaneh N.K. (1990) Biological dosimeters, in: Biochemistry of ionizing radiations, Raven Press, New York, pp. 160-175.

Wodnar-Filipowicz A., Lyman S.D., Grathwohl A., Tichelli A., Speck B., Nissen C. (1996) Flt3 ligand level reflects hematopoietic progenitor cell function in aplastic aemia and chemotherapyinduced bone marrow aplasia, Blood 88, 4493-4499.

Zoetelief J., Wagemaker G., Broerse J.J. (1998) Dosimetry for total body irradiation of rhesus monkeys with $300 \mathrm{kV}$ X-rays, Intern. J. Rad. Biol. 74, 265-272. 